OPEN ACCESS

Edited by: Kimberly Huey,

Drake University, USA

Reviewed by:

Brandt D. Pence,

University of Memphis, USA

Rudy Valentine,

lowa State University, USA

${ }^{*}$ Correspondence:

Tomas Venckunas

Tomas.Venckunas@/su.It

Specialty section:

This article was submitted to

Exercise Physiology,

a section of the journal

Frontiers in Physiology

Received: 16 November 2016 Accepted: 05 January 2017 Published: 23 January 2017

Citation:

Kvedaras $M$, Minderis P, Fokin A,

Ratkevicius $A$, Venckunas $T$ and

Lionikas A (2017) Forced Running

Endurance Is Influenced by Gene(s) on

Mouse Chromosome 10

Front. Physiol. 8:9.

doi: 10.3389/fphys.2017.00009

\section{Forced Running Endurance Is Influenced by Gene(s) on Mouse Chromosome 10}

\author{
Mindaugas Kvedaras ${ }^{1}$, Petras Minderis ${ }^{1}$, Andrej Fokin ${ }^{1}$, Aivaras Ratkevicius ${ }^{1}$, \\ Tomas Venckunas ${ }^{1 *}$ and Arimantas Lionikas ${ }^{2}$ \\ ${ }^{1}$ Institute of Sport Science and Innovations, Lithuanian Sports University, Kaunas, Lithuania, ${ }^{2}$ School of Medicine, Medical \\ Sciences and Nutrition, College of Life Sciences and Medicine, University of Aberdeen, Aberdeen, UK
}

Phenotypic diversity between laboratory mouse strains provides a model for studying the underlying genetic mechanisms. The $\mathrm{A} / \mathrm{J}$ strain performs poorly in various endurance exercise models. The aim of the study was to test if endurance capacity and contractility of the fast- and slow-twitch muscles are affected by the genes on mouse chromosome 10. The C57BL/6J (B6) strain and C57BL/6J-Chr 10 A/J/NaJ (B6.A10) consomic strain which carries the $\mathrm{A} / \mathrm{J}$ chromosome 10 on a $\mathrm{B} 6$ strain background were compared. The B6.A10 mice compared to B6 were larger in body weight $(p<0.02): 27.2 \pm 1.9$ vs. $23.8 \pm 2.7$ and $23.4 \pm 1.9$ vs. $22.9 \pm 2.3 \mathrm{~g}$, for males and females, respectively, and in male soleus weight $(p<0.02): 9.7 \pm 0.4$ vs. $8.6 \pm 0.9 \mathrm{mg}$. In the forced running test the B6.A10 mice completed only $64 \%$ of the B6 covered distance $(p<0.0001)$. However, there was no difference in voluntary wheel running $(p=0.6)$ or in fatigability of isolated soleus $(p=0.24)$ or extensor digitorum longus (EDL, $p=0.7$ ) muscles. We conclude that chromosome 10 of the $\mathrm{A} / \mathrm{J}$ strain contributes to reduced endurance performance. We also discuss physiological mechanisms and methodological aspects relevant to interpretation of these findings.

\section{Keywords: electric stimulation, specific force, chromosome substitution strains, exercise, skeletal muscle}

\section{INTRODUCTION}

Endurance as a component of physical fitness is an important determinant of health and well-being. For instance, the risk of mortality due to cardiovascular disease in obese men is significantly reduced with increasing level of fitness (Lee et al., 1999). Genetic factors account for a substantial portion of endurance capacity with heritability estimates around 50\% (Bouchard et al., 1998). Hence, understanding of the underlying genetics might reveal new biomarkers and targets for pharmaceutical interventions to improve fitness and health.

The ability to generate energy via aerobic pathways is a significant determinant of endurance performance. It has been demonstrated that endurance improvement is concomitant with an increase in $\mathrm{VO}_{2} \max$ and activity of the mitochondrial enzymes (Vollaard et al., 2009). Indeed, genes involved in aerobic oxidation and mitochondrial biogenesis may have a substantial influence on endurance capacity. For example, overexpression of PPARdelta (Wang et al., 2004), which stimulates mitochondrial biogenesis in skeletal muscle, or PEPCK-C (Hakimi et al., 2007), which can contribute to the flux through the tricarboxylic acid (TCA) cycle (Burgess et al., 2004), 
significantly improved running endurance in mice. However, it remains unclear whether these genes play a role in variability in endurance observed in human populations or animal models.

Analyses of the laboratory mouse strains revealed that genetic variability can substantially influence endurance capacity. This influence is evident even when different types of motor activity are explored. For instance, the A/J strain performs poorly in forced running (Lightfoot et al., 2001; Hoit et al., 2002; Courtney and Massett, 2012) and swimming (Kilikevicius et al., 2013) endurance tests compared to other strains including the C57BL/6J (B6). Thus, these two strains provide an interesting model for studying the genetic mechanisms influencing endurance performance.

Chromosome substitution strains, also known as consomic strains, provide a simple model for exploring genetic influences. They consist of a panel of strains where one chromosome of a host strain is replaced by a homologous chromosome of a donor strain (Matin et al., 1999; Singer et al., 2004; Ishii et al., 2011). Phenotypic differences between host and a consomic strain implicate the variants of genes residing on a particular chromosome in their etiology. A genome wide screening of a panel of consomic strains would still require a substantial number of animals (Shao et al., 2008). However, it offers a cost effective model for preliminary hypothesis testing in the instances where analyses can be restricted to a limited number of chromosomes (Chr).

The C57BL/6J-Chr $10^{\mathrm{A} / \mathrm{J}} / \mathrm{NaJ}$ (B6.A10) consomic strain carrying $\mathrm{A} / \mathrm{J} \mathrm{Chr} 10$ on the $\mathrm{B} 6$ strain background provides a useful model for studying the genetic mechanisms contributing to impaired endurance capacity of the $\mathrm{A} / \mathrm{J}$ strain. A gene encoding the key enzyme of the TCA cycle, citrate synthase (Cs), resides on mouse Chr 10. Both human (Vollaard et al., 2009) and rodent (Holloszy, 1967) studies demonstrated that endurance training is accompanied by an increase in CS activity. Such adaptive response implies that $C$ s may play a limiting role in endurance capacity. Furthermore, A/J mice carry a strain-specific allele of the gene. We have reported that enzymatic activity of CS in skeletal muscle samples from the $\mathrm{A} / \mathrm{J}$ strain is $\sim 50 \%$ lower compared to B6 allele (Ratkevicius et al., 2010). As an initial step to investigating the role of the genes residing on Chr 10 in endurance capacity of the A/J mice, we used the B6.A10 consomic strain to explore relevant phenotypes.

The aim of this study was to test the hypothesis that running endurance and skeletal muscle resistance to fatigue may be reduced in the B6.A10 strain.

\section{MATERIALS AND METHODS}

\section{Animals}

All animal procedures were approved by the Lithuanian State Food and Veterinary Service (Ref. \# 0230). Breeding nuclei of the C57BL/6J (B6) and C57BL/6J-Chr 10 A/J/NaJ (B6.A10) strains were purchased from Charles River and the Jackson Laboratory, respectively. Samples of the experimental animals were bred locally.

Mice were housed in standard cages, one to three same sex animals per cage, ambient temperature $20-21^{\circ} \mathrm{C}$ and $40-60$
$\%$ humidity, with 12-h light/ 12-h dark cycle. Animals were fed standard chow diet (LabDiet 5001) and received tap water ad libitum. The forced running experimental procedure on 16 B6 ( 8 females and 8 males) and 21 B6.A10 (7 and 14, respectively) was initiated at 12-13 week of age. Voluntary wheel running was measured in separate group of 10 B6 and 10 B6.A10 males at 1516 week of age. The ex vivo testing was carried out on a separate group of 6 B6 and 6 B6.A10 males of 18-19 week of age.

\section{Forced Treadmill Running Experiment}

Animals were tested on a 5-lane treadmill (LE8710MAP, Panlab, Harvard Apparatus, SL, Spain) with an exercise area of $37 \times 5 \times$ $5 \mathrm{~cm}(\mathrm{~L} \times \mathrm{W} \times \mathrm{H})$ in each lane, and with a built-in air puff stimulation at the end of the lane. All treadmill sessions were carried out during the dark cycle under red light illumination. Each exposure to the treadmill began with a 2-min habituation period on a stationary treadmill belt at a zero incline. Two habituation sessions of 15 min were carried out on 2 consecutive days prior to endurance test. During those sessions mice ran on the treadmill at a speed of $16.8 \mathrm{~m} / \mathrm{min}$ at zero incline. The treadmill belt was cleaned with $70 \%$ ethanol between the groups.

Two days after completion of habituation mice were subjected to an endurance test. The test is a modification of the protocol described earlier (Knab et al., 2009; Massett et al., 2009). The starting speed of the treadmill at a zero incline was set at 16.8 $\mathrm{m} / \mathrm{min}$. The speed was increased by $3 \mathrm{~m} / \mathrm{min}$ every $2 \mathrm{~min}$ until $40.8 \mathrm{~m} / \mathrm{min}$ speed was reached. If a $2 \mathrm{~min}$ stage of $40.8 \mathrm{~m} / \mathrm{min}$ speed was successfully accomplished, the speed of $40.8 \mathrm{~m} / \mathrm{min}$ was maintained until exhaustion. The endurance capacity was characterized by the distance ran over the test.

Animals were motivated to run by air puffs. It was activated when the mouse touched the grid at the end of lane. If the mouse still refused to run then it was encouraged to continue running by gentle hand prodding of the tail or hindquarters. The mice readily responded to this stimulus as has been described by Steiner et al. (2011). The point of exhaustion was defined as the time (in min) at which the mouse could no longer keep pace with the treadmill despite of delivered air puffs and continuous (lasting up to $10 \mathrm{~s}$ ) hand prodding.

\section{Voluntary Wheel Running}

Animals were single housed in a cage equipped with a running wheel of $71.6 \mathrm{~cm}$ in inner circumference (Tecniplast, Italy). Following 1 day habituation period running wheel activity was recorded as revolutions of the wheel over 3 consecutive days. This permitted calculation of the distance ran over that period of time. However, it was not possible to determine the actual time spent on the wheel.

\section{Contractile Properties of Fast- and Slow-Twitch Muscles}

Contractility of extensor digitorum longus (EDL) and soleus muscles was examined. All procedures were carried out at room temperature $\left(23-25^{\circ} \mathrm{C}\right)$. Following sacrifice, soleus and EDL muscles of randomly selected hind limb were excised for contractile measurements. Sutures were attached to the proximal and distal tendons of isolated muscles. Thereafter muscle was 
mounted between two platinum plate electrodes in $100 \mathrm{~mL}$ tissue bath (Radnoti, USA) filled with Tyrode's solution (in mM: 121 $\mathrm{NaCl}, 5 \mathrm{KCl}, 0.5 \mathrm{MgCl}_{2}, 1.8 \mathrm{CaCl}_{2}, 0.4 \mathrm{NaH}_{2} \mathrm{PO}_{4}, 0.1 \mathrm{NaEDTA}$,

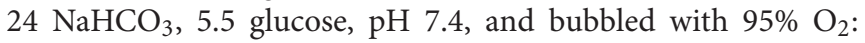
$5 \% \mathrm{CO}_{2}$ ). The distal tendon was attached to a stable hook and the proximal end was tied directly to the lever of the muscle test system (1200A-LR Muscle Test System, Aurora Scientific Inc., Canada). Both soleus and EDL of the same hind limb were processed in an alternating order between the animals. While processing the first muscle the other was kept in a bath with Tyrode solution. Mounted muscle was left to equilibrate in the solution for $10 \mathrm{~min}$. Afterwards the muscle was stimulated using a custom-made stimulator by $25 \mathrm{~V}$ square pulses. It has been determined in a pilot experiment that this voltage elicits maximal contraction. Muscle length was increased and twitch force measured every $30 \mathrm{~s}$ until no further increase in force was observed. Muscle was kept at this optimal length $\left(\mathrm{L}_{0}\right)$ during the subsequent experiments. Muscles were photographed with a scale in the background in order to assess muscle length with a precision of $0.1 \mathrm{~mm}$. Twitch contraction time (CT) was assessed as the time elapsed from the beginning of the contraction to its peak. Twitch half relaxation time (HRT) was measured as the time taken for the force to decline to $50 \%$ of the peak value. At $\mathrm{L}_{0}$ muscle was subjected to $300 \mathrm{~ms}$ (EDL) or $900 \mathrm{~ms}$ (soleus) trains of pulses of increasing frequencies $(20,50,80,100,150,200 \mathrm{~Hz})$. Maximal isometric force $\left(\mathrm{P}_{0}\right)$ was estimated from plateau in force-frequency curve and usually occurred at $100 \mathrm{~Hz}$ for SOL and at $100-150 \mathrm{~Hz}$ for EDL.

Following the force-frequency procedure muscle was subjected to a fatigue protocol consisting of 180 repeated isometric contractions at $40 \mathrm{~Hz}$. A train of stimuli $(250 \mathrm{~ms}$ in duration for $\mathrm{EDL}$, or $500 \mathrm{~ms}$ for SOL) were delivered every second for EDL and every $1.1 \mathrm{~s}$ for SOL, respectively. Thus, duration of fatigue protocol for EDL and SOL was 180 and $198 \mathrm{~s}$, respectively. The fatigue index was calculated as the ratio between the force of the final and the first contraction of the fatigue protocol multiplied by 100 .

After the measurements, the muscle was cleaned from all visible tendons, blotted, and weighed on an analytical balance (Kern, ABT 320-4M, Germany). Muscle physiological crosssectional area (CSA) was calculated by dividing wet muscle mass by the optimal fiber length $\left(\mathrm{L}_{\mathrm{f}}\right)$ and the density of mammalian skeletal muscle: CSA $\left(\mathrm{mm}^{2}\right)=$ mass $(\mathrm{mg}) /$ length $(\mathrm{mm}) / 1.06$ $\left(\mathrm{mg} / \mathrm{mm}^{3}\right)$ (Brooks and Faulkner, 1988). $\mathrm{L}_{\mathrm{f}}$ was calculated from the $\mathrm{L}_{\mathrm{f}} / \mathrm{L}_{0}$ ratio of 0.70 or 0.45 for soleus and $\mathrm{EDL}$, respectively (Brooks and Faulkner, 1988). The specific tension $\left(\mathrm{sP}_{0}\right)$ was determined by the ratio between $\mathrm{P}_{0}$ and CSA.

\section{CS Enzyme Activity}

CS activity was measured as previously described (Ratkevicius et al., 2010). The gastrocnemius muscle samples from the B6 $(n=9)$ and B6.A10 $(n=9)$ males were homogenized in icecold lysis buffer ( $50 \mathrm{mM}$ Tris. $\mathrm{HCl}, 1 \mathrm{mM}$ EDTA, $1 \mathrm{mM}$ EGTA, $1 \%$ Triton $\mathrm{X}-100, \mathrm{pH}$ was adjusted to 7.0 ) with an ULTRATURRAX homogenizer (Rose Scientific, Edmonton, Canada). Following shaking for $60 \mathrm{~min}$ the homogenates were centrifuged at $13,000 \mathrm{~g}$ at $4^{\circ} \mathrm{C}$ for $10 \mathrm{~min}$ and the protein concentration was measured in the supernatant using the Bradford assay (Bio-Rad, Hertfordshire, UK). The reaction reagent consisted of $100 \mathrm{mM}$ triethanolamine-HCl, DTNB $(100 \mu \mathrm{M}), 0.25 \%$ Triton$\mathrm{X}$ (vol/vol), $0.5 \mathrm{mM}$ oxaloacetate, $0.31 \mathrm{mM}$ acetyl CoA with $\mathrm{pH}$ adjusted to 8.0. Ten microliters of muscle homogenate was added to start the reaction in $1000 \mu \mathrm{L}$. The molar extinction coefficient of $13,600 \mathrm{M}^{-1} \cdot \mathrm{cm}^{-1}$ was used to assess the maximum CS activity (Vmax) at $412 \mathrm{~nm}$ during the first 2 min of the reaction. The assay was carried out at room temperature $\left(\sim 21^{\circ} \mathrm{C}\right)$, and $\mathrm{CS}$ from porcine heart was used as a standard (C3260-200UN, SigmaAldrich, UK) for assay calibration.

\section{Statistical Analyses}

A 2-way ANOVA (Strain and Sex), 2-way ANCOVA (body weight as covariate) and Mann-Whitney $U$-test were performed where appropriate using IBM SPSS Statistics (v21) software. Mean and $S D$ is presented unless stated otherwise.

\section{RESULTS}

\section{Endurance Capacity}

First we aimed at determining if consomic strain carrying Chr 10 of $\mathrm{A} / \mathrm{J}$ strain would show a reduced performance in the forced running endurance test.

The B6 strain showed a superior endurance capacity by running significantly further compared to the B6.A10 strain, $391 \pm 53$ vs. $251 \pm 54 \mathrm{~m}$ respectively $(p<0.0001)$. There was no sex effect $(p=0.6)$ on endurance performance (Figure 1A). Although the B6.A10 mice were heavier $(p<0.02)$ compared to the B6; $27.2 \pm 1.9$ vs. $23.8 \pm 2.7$ and $23.4 \pm 1.9$ vs. $22.9 \pm 2.3 \mathrm{~g}$, for males and females, respectively, the strain effect on forced running distance remained statistically significant $(p<0.0001)$ after inclusion of body weight as covariate.

\section{Voluntary Wheel Running}

We then wanted to examine if the difference in forced running endurance would be paralleled by a strain effect on voluntary activity.

Voluntary running capacity was assessed in male mice over the course of $72 \mathrm{~h}$. Only males were studied because no sex effect was noted in the forced running test. The tested animals ran $2649 \pm 996 \mathrm{~m}$ per day. There was no statistically significant difference between the B6 and B6.A10 strains ( $p=0.6$; Figure 1B).

\section{Contractile Properties of Isolated Muscles}

To test the hypothesis that peripheral mechanisms of fatigue may be contributing to poor performance in the forced running trial we analyzed contractility of isolated fast- and slow-twitch muscle of the B6 and B6.A10 males. Only males were studied because no sex effect was noted in the forced running.

Gastrocnemius weight as well as EDL weight, length, and CSA were similar between the strains, whereas soleus weight and the CSA were slightly, yet statistically significantly, larger $(p<0.05)$ in B6.A10 compared to the B6 strain (Table 1).

The peak force of isometric contraction was similar between the B6 and B6.A10 strains in both soleus, $192 \pm 24$ vs. 

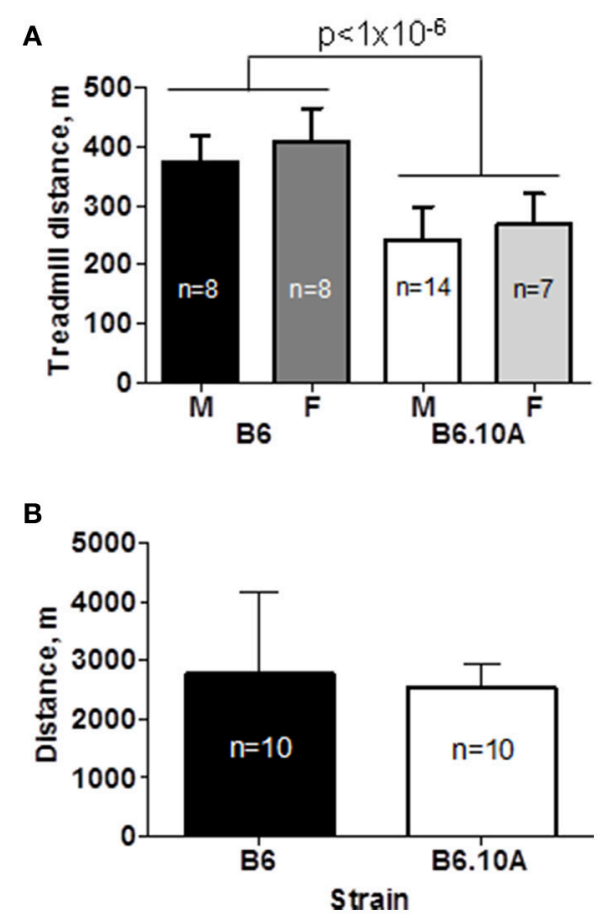

FIGURE 1 | Chromosome 10 of the A/J strain confers lower endurance capacity. (A) Forced treadmill running distance of the consomic B6.A10 strain was reduced compared to the host C57BL/6 (B6) strain. Males (M) and females (F) were similarly affected. (B) Voluntary wheel running distance over $24 \mathrm{~h}$ period was not different between males of the two strains. Mean and $S D$ shown; $n$ indicates number of animals per group; $p$-value for weight adjusted strain effect is shown.

TABLE 1 | Properties of skeletal muscle samples of B6 and B6.A10 strain males. Mean $\pm S D$.

\begin{tabular}{|c|c|c|c|}
\hline & $\mathrm{B} 6(n=6)$ & B6.A10 $(n=6)$ & $p$-value* \\
\hline BW (g) & $28.7 \pm 2.0$ & $29.1 \pm 1.0$ & 0.699 \\
\hline Gastrocnemius weight (mg) & $129.8 \pm 7.8$ & $138.8 \pm 5.4$ & 0.065 \\
\hline Soleus weight (mg) & $8.6 \pm 0.9$ & $9.7 \pm 0.4$ & 0.015 \\
\hline Soleus Lo (mm) & $13.9 \pm 0.6$ & $14.1 \pm 0.5$ & 0.485 \\
\hline Soleus $L_{f}(\mathrm{~mm})$ & $9.9 \pm 0.4$ & $10.0 \pm 0.4$ & 0.589 \\
\hline Soleus CSA (mm²) & $0.9 \pm 0.1$ & $1.0 \pm 0.1$ & 0.041 \\
\hline EDL (mg) & $10.5 \pm 1.2$ & $11.1 \pm 0.6$ & 0.394 \\
\hline EDL Lo (mm) & $15.9 \pm 0.5$ & $15.9 \pm 0.7$ & 0.699 \\
\hline$E D L L_{f}(m m)$ & $7.2 \pm 0.2$ & $7.2 \pm 0.3$ & 0.699 \\
\hline EDL CSA $\left(\mathrm{mm}^{2}\right)$ & $1.6 \pm 0.2$ & $1.6 \pm 0.0$ & 0.818 \\
\hline
\end{tabular}

*Mann-Whitney U-test between the strains, exact significance displayed; values $<0.05$ are highlighted in bold. BW, body weight; Lo, optimal length; $L_{f}$, optimal fiber length; CSA, physiological cross-sectional area; $n$ indicates number of samples tested.

$211 \pm 10 \mathrm{mN}(p=0.13)$, respectively, and EDL muscles, $209 \pm 21$ vs. $217 \pm 13 \mathrm{mN}$ ( $p=0.59)$. The isometric contraction force after adjustment for the CSA of the muscle did not differ between the strains in either soleus or EDL muscle (Figure 2A). The temporal properties of twitch contraction and relaxation did not differ between the strains either (Figure 2B). A series of repeated
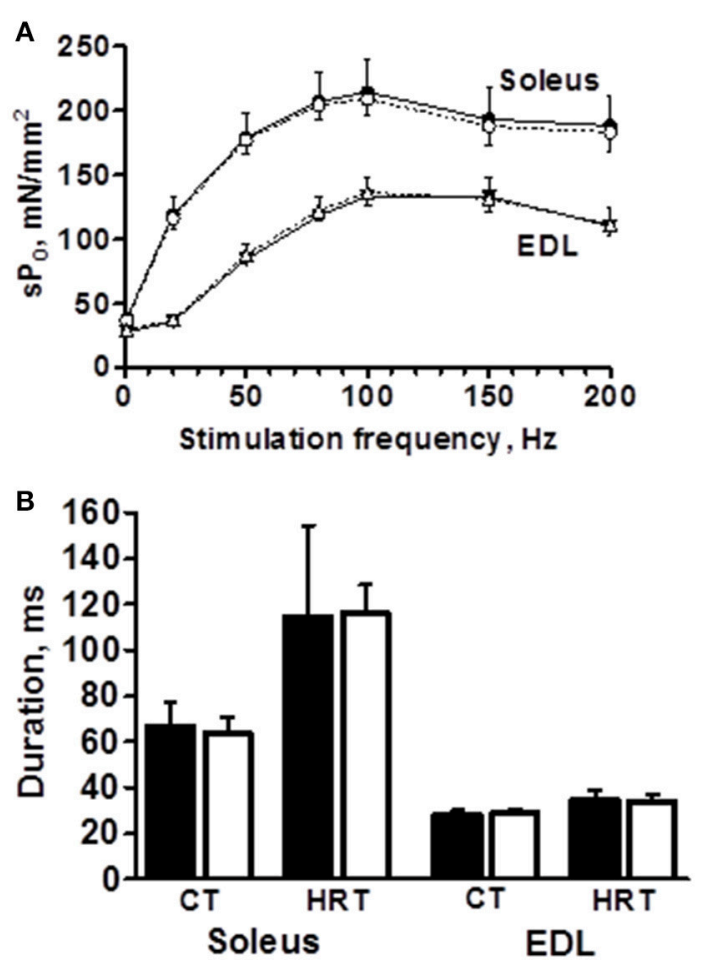

FIGURE 2 | Contractile properties of isolated slow- (soleus) and fast-twitch (extensor digitorum longus, EDL) muscles are not associated with chromosome $\mathbf{1 0}$ genotype. Force-frequency relationship expressed as specific force (A), contraction (CT) and half relaxation times $(\mathrm{HRT})$ of single twitch $\mathbf{( B )}$ are shown. Black symbols/bars represent B6 and white B6.A10 strain. Mean and SD shown.

contractions in both fast- and slow-twitch muscle resulted in a similar level of fatigue between the strains (Figure 3). Fatigue index in B6 and B6.A10 soleus was $34 \pm 5$ and $31 \pm 3 \%$, respectively $(p=0.24)$, and $27 \pm 2$ and $27 \pm 4 \%(p=0.7)$ in the EDL.

\section{CS Enzymatic Activity}

The CS activity in gastrocnemius muscle was assessed to examine if it could have contributed to reduced forced running endurance of the B6.A10 strain. The analysis indicated that CS activity was significantly lower $(p<0.0001)$ in B6.A10 compared to B6 strain, $487.7 \pm 69.5$ vs. $754.0 \pm 68.6 \mathrm{U} / g$, respectively.

\section{DISCUSSION}

The main findings of this study are that Chr 10 of the A/J strain is associated with substantially lower endurance capacity compared to the B6 strain. The between strain difference remained robust and highly significant even following adjustments for body weight. Consistently with our recent observation in a separate study involving the same strains (Kilikevicius et al., 2016), it also emerged that soleus muscle weight was higher in B6.A10 compared to the $\mathrm{B} 6$ mice. 

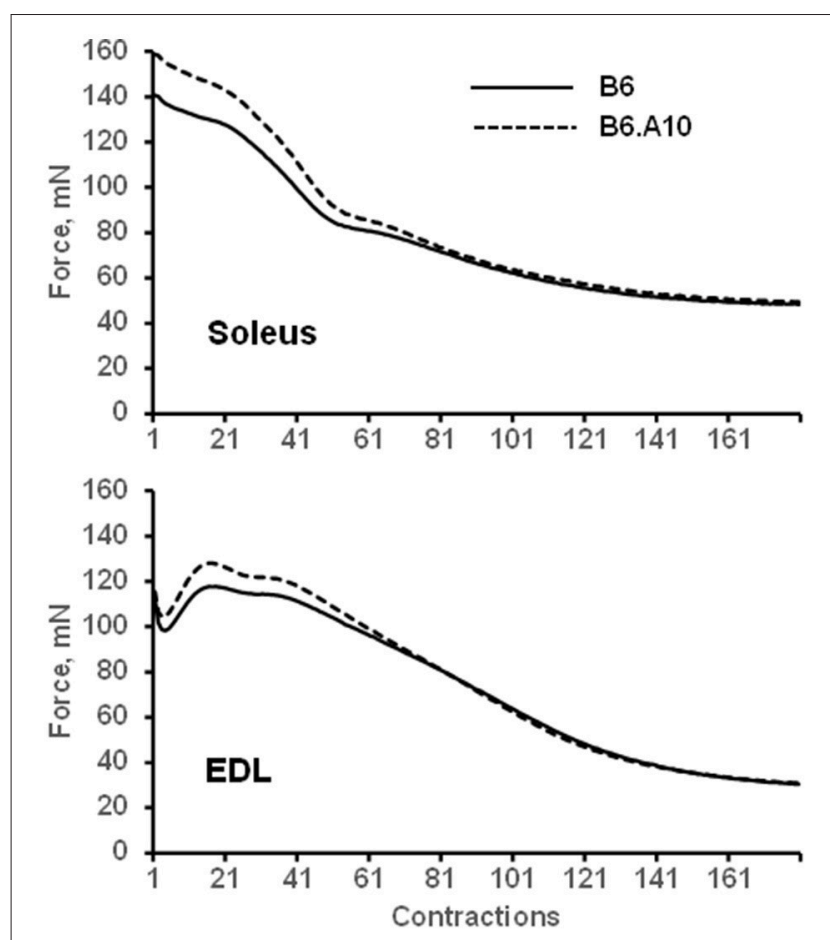

FIGURE 3 | Fatigue of isolated muscle is not linked to chromosome 10 genotype. Mean force of 180 repeated contractions of slow-twitch soleus (top) and fast-twitch EDL (bottom) muscles are shown. Solid and dashed lines represent B6 and B6.A10 strains, respectively.

Endurance capacity of the B6 strain measured in the present study as the distance run during the test was comparable to values in the literature despite the differences in the testing protocol and age (Massett, 2013; Courtney and Massett, 2014). In these earlier studies endurance capacity of the A/J strain equaled to just 43\% of that in the B6 strain. Our model, showing that B6.A10 mice ran only $64 \%$ of the distance completed by B6, implicates $\mathrm{Chr}$ 10 gene(s) in impaired endurance of the A/J strain. This novel finding adds to the previous knowledge of the role of Chr 14; specifically, males of consomic strain B6.A14 ran $\sim 84 \%$ of the distance completed by B6 strain (Courtney and Massett, 2014). Thus, it emerges that the difference in endurance between the B6 and $\mathrm{A} / \mathrm{J}$ strains is polygenic in nature, caused by chromosome 10 and 14 genes; the identity of the causative genes, however, remains to be determined.

Physical activity is an important determinant of health and fitness. Voluntary wheel running is extensively used for quantification of physical activity in rodents. The majority of rodent species avidly engage in this locomotor activity although it is debatable what motivates animals to run (Careau et al., 2012). Although both voluntary and forced running represent locomotor activities, mechanisms limiting performance under these two conditions are likely to differ. The forced running test is continuous and limited by the neuromuscular and/or cardiovascular systems, while the voluntary running consists of bursts of activity accumulated over a long period of time and hence is not determined by the same physiological mechanisms.
Such a notion is consistent with the observations that voluntary running distance did not differ markedly between the B6 and A/J strains (Lightfoot et al., 2010; Courtney and Massett, 2012). A similar uncoupling of performance in the two types of activity was also observed between other strains; for instance 129 S1/SvImJ mice performed poorly in voluntary running compared to the B6 but matched their performance in forced running (Lightfoot et al., 2010; Courtney and Massett, 2012). Thus, mechanisms limiting forced running endurance in the B6.A10 strain do not affect voluntary running of these animals.

Enzymatic activity of citrate synthase (CS) has been used as a biomarker of mitochondrial content (Larsen et al., 2012) and function (Jacobs et al., 2013) in skeletal muscle. It has been known that endurance exercise training leads to an increase in CS activity in exercised skeletal muscles (Holloszy and Booth, 1976). The CS encoding gene, Cs, resides in the telomeric region of mouse Chr 10. The A/J strain is characterized by $\sim 50 \%$ reduced enzymatic activity of CS in skeletal muscle (Ratkevicius et al., 2010). Consistently with that, albeit a smaller reduction of $\sim 35 \%$ was observed in the present study in B6.A10 strain carrying the A/J strain Chromosome 10. This discrepancy between the A/J and B6.A10 strains suggests that $\sim 35 \%$ reduction can be attributed to Chromosome 10, whereas the remaining difference is due to the mechanisms governed by the factors out with Chr 10, which could affect quantity of the enzyme in the muscle (e.g., by influencing proportion of oxidative fibers) rather than the innate CS activity.

We hypothesized that the impaired endurance capacity of the B6.A10 strain might be a reflection of fatigue resistance of skeletal muscles. However, fatigability of the muscles was not strain dependent. To understand this unexpected observation we first considered whether sample size could have been too small. That, however, is unlikely. If the strain effect on the fatigue index of isolated muscle was proportional to that on the forced running endurance, we had statistical power $>85 \%$ for detecting it at alpha level of $1 \%$ in this sample. The second possible explanation is methodological. It has been demonstrated that isolated muscles fatigue faster compared to individual muscle fibers dissected from the same muscle because of the restricted oxygen diffusion to the core of the muscle (Zhang et al., 2006). If differences in the aerobic metabolism of skeletal muscle were responsible for accelerated fatigue in the forced running test (a plausible hypothesis considering the difference in CS activity between the B6 and B6.A10), its relevance might have been substantially diminished under the hypoxic conditions where contractility would shift toward reliance on anaerobic metabolism. Examining fatigue properties in isolated muscle fibers and/or the whole muscle in situ would be required to address this question. The third alternative explanation is that forced running endurance could be primarily limited by cardiovascular function. The echocardiographic variables (ventricular wall thickness, chamber size) in the B6 strain exhibit properties reminiscent of the "athlete's heart" in comparison to A/J (Hoit et al., 2002). The estimates of the cardiac output based on the systolic-diastolic volume difference and heart rate are $\sim 15 \%$ lower in the A/J strain compared to the B6 (Hoit et al., 2002; Lake et al., 2009; The Jackson Laboratory, 2010). However, as ventricular parameters of 
the B6.A10 strain are not known, its direct comparison to the B6 strain is not possible. Further studies will be required to prioritize between the contribution of cardiac and muscular mechanisms to the difference in fatigability between the B6 and B6.A10 mice.

A difference in soleus muscle weight between the B6 and B6.A10 strains (Table 1) provides further support to recently reported observation between the same strains (Kilikevicius et al., 2016). Albeit a $\sim 13 \%$ increase in soleus of B6.A10 strain is less extensive than $\sim 40 \%$ reported in that study, collectively these findings are consistent with a notion that $\mathrm{A} / \mathrm{J}$ variant of one or more chromosome 10 genes confer an increase in soleus weight. The direction of the effect, i.e., B6.A10 > B6, is somewhat surprising because in the B6 vs. A/J comparison the former exhibits higher weight due to more numerous fibers (Kilikevicius et al., 2013). The contractile function measured in the present study was comparable with literature, both for the soleus (Brooks and Faulkner, 1988) and EDL (Amthor et al., 2007) muscles. In general, there was no difference between the B6 and B6.A10 strains in contraction and relaxation times (Figure 2), although peak isometric force in B6.A10 soleus was slightly elevated mirroring muscle weight difference between the strains. The mechanisms and gene(s) underlying the strain difference remain to be determined.

\section{REFERENCES}

Amthor, H., Macharia, R., Navarrete, R., Schuelke, M., Brown, S. C., Otto, A., et al. (2007). Lack of myostatin results in excessive muscle growth but impaired force generation. Proc. Natl. Acad. Sci. U.S.A. 104, 1835-1840. doi: 10.1073/pnas.0604893104

Bouchard, C., Daw, E. W., Rice, T., Perusse, L., Gagnon, J., Province, M. A., et al. (1998). Familial resemblance for VO2max in the sedentary state: the HERITAGE family study. Med. Sci. Sports Exerc. 30, 252-258. doi: 10.1097/0000 5768-199802000-00013

Brooks, S. V., and Faulkner, J. A. (1988). Contractile properties of skeletal muscles from young, adult and aged mice. J. Physiol. 404, 71-82. doi: 10.1113/jphysiol.1988.sp017279

Burgess, S. C., Hausler, N., Merritt, M., Jeffrey, F. M., Storey, C., Milde, A., et al. (2004). Impaired tricarboxylic acid cycle activity in mouse livers lacking cytosolic phosphoenolpyruvate carboxykinase. J. Biol. Chem. 279, 48941-48949. doi: 10.1074/jbc.M407120200

Careau, V., Bininda-Emonds, O. R., Ordonez, G., and Garland, T. Jr., (2012). Are voluntary wheel running and open-field behavior correlated in mice? Different answers from comparative and artificial selection approaches. Behav. Genet. 42, 830-844. doi: 10.1007/s10519-012-9543-0

Courtney, S. M., and Massett, M. P.,(2012). Identification of exercise capacity QTL using association mapping in inbred mice. Physiol. Genomics 44, 948-955. doi: 10.1152/physiolgenomics.00051.2012

Courtney, S. M., and Massett, M. P. (2014). Effect of chromosome substitution on intrinsic exercise capacity in mice. F1000Res. 3, 9. doi: 10.12688/f1000 research.3-9.v1

Hakimi, P., Yang, J., Casadesus, G., Massillon, D., Tolentino-Silva, F., Nye, C. K., et al. (2007). Overexpression of the cytosolic form of phosphoenolpyruvate carboxykinase (GTP). in skeletal muscle repatterns energy metabolism in the mouse. J. Biol. Chem. 282, 32844-32855. doi: 10.1074/jbc.M706127200

Hoit, B. D., Kiatchoosakun, S., Restivo, J., Kirkpatrick, D., Olszens, K., Shao, H., et al. (2002). Naturally occurring variation in cardiovascular traits among inbred mouse strains. Genomics 79, 679-685. doi: 10.1006/geno.2002.6754

Holloszy, J. O. (1967). Biochemical adaptations in muscle. Effects of exercise on mitochondrial oxygen uptake and respiratory enzyme activity in skeletal muscle. J. Biol. Chem. 242, 2278-2282.
In conclusion, $\mathrm{Chr} 10$ of the $\mathrm{A} / \mathrm{J}$ strain contributes to the reduced CS enzymatic activity and endurance performance. The effect may be mediated by the cardiovascular function, although the role of skeletal muscle cannot be ruled out. An intercross between the B6 and $\mathrm{B} 6.10 \mathrm{~A}$ strains provides an attractive research model for further analyses and identification of gene(s) determining endurance capacity.

\section{AUTHOR CONTRIBUTIONS}

$\mathrm{AL}, \mathrm{AR}$, and TV designed the study; $\mathrm{MK}$ carried out forced running and voluntary wheel running experiments with supervision of TV; PM performed physiological analyses of isolated muscles; AF measured enzyme activity; $\mathrm{AL}$ and $\mathrm{MK}$ wrote the manuscript with contribution of all authors.

\section{ACKNOWLEDGMENTS}

The authors wish to acknowledge technical assistance from Mrs. Indre Libnickienè and intellectual input from Dr. David A. Blizard. This research was funded by the European Social Fund under the Global Grant measure. Grant VP1-3.1-ŠMM-07-K-02057 was awarded to AL.

Holloszy, J. O., and Booth, F. W. (1976). Biochemical adaptations to endurance exercise in muscle. Annu. Rev. Physiol. 38, 273-291. doi: 10.1146/annurev. ph.38.030176.001421

Ishii, A., Koide, T., Takahashi, A., Shiroishi, T., Hettinger, T. P., Frank, M. E., et al. (2011). B6-MSM consomic mouse strains reveal multiple loci for genetic variation in sucrose octaacetate aversion. Behav. Genet. 41, 716-723. doi: 10.1007/s10519-011-9464-3

Jacobs, R. A., Díaz, V., Meinild, A. K., Gassmann, M., and Lundby, C. (2013). The C57Bl/6 mouse serves as a suitable model of human skeletal muscle mitochondrial function. Exp. Physiol. 98, 908-921. doi: 10.1113/ expphysiol.2012.070037

Kilikevicius, A., Bunger, L., and Lionikas, A. (2016). Baseline muscle mass is a poor predictor of functional overload-induced gain in the mouse model. Front. Physiol. 15:534. doi: 10.3389/fphys.2016.00534

Kilikevicius, A., Venckunas, T., Zelniene, R., Carroll, A. M., Lionikaite, S., Ratkevicius, A., et al. (2013). Divergent physiological characteristics and responses to endurance training among inbred mouse strains. Scand. J. Med. Sci. Sports 23, 657-668. doi: 10.1111/j.1600-0838.2012. 01451

Knab, A. M., Bowen, R. S., Moore-Harrison, T., Hamilton, A. T., Turner, M. J., and Lightfoot, J. T. (2009).Repeatability of exercise behaviors in mice. Physiol. Behav. 98, 433-440. doi: 10.1016/j.physbeh.2009.07.006

Lake, J., Donahue, L., and Davisson, M.T. (2009). Multi-System Analysis of Mouse Physiology, C57BL/6J-Chr\#A/J/NaJ Chromosome Substitution Panel. MPD:19940. Mouse Phenome Database Web Site. Bar Harbor, ME: The Jackson Laboratory.

Larsen, S., Nielsen, J., Hansen, C. N., Nielsen, L. B., Wibrand, F., Stride, N., et al. (2012). Biomarkers of mitochondrial content in skeletal muscle of healthy young human subjects. J. Physiol. 590, 3349-3360. doi: 10.1113/ jphysiol.2012.230185

Lee, C. D., Blair, S. N., and Jackson, A. S. (1999). Cardiorespiratory fitness, body composition, and all-cause and cardiovascular disease mortality in men. Am. J. Clin. Nutr. 69, 373-380.

Lightfoot, J. T., Leamy, L., Pomp, D., Turner, M. J., Fodor, A. A., Knab, A., et al. (2010). Strain screen and haplotype association mapping of wheel running in inbred mouse strains. J. Appl. Physiol. 109, 623-634. doi: 10.1152/japplphysiol.00525.2010 
Lightfoot, J. T., Turner, M. J., Debate, K. A., and Kleeberger, S. R. (2001). Interstrain variation in murine aerobic capacity. Med. Sci. Sports Exerc. 33, 2053-2057. doi: 10.1097/00005768-200112000-00012

Massett, M. P. (2013). Exercise Capacity in 34 Inbred Strains of Mice. MPD:46503. Mouse Phenome Database Web Site. Bar Harbor, ME: The Jackson Laboratory.

Massett, M. P., Fan, R., and Berk, B. C. (2009). Quantitative trait loci for exercise training responses in FVB/NJ and C57BL/6J mice. Physiol. Genomics 40, 15-22. doi: 10.1152/physiolgenomics.00116.2009

Matin, A., Collin, G. B., Asada, Y., Varnum, D., and Nadeau, J. H. (1999). Susceptibility to testicular germ-cell tumours in a 129.MOLF-Chr 19 chromosome substitution strain. Nat. Genet. 23, 237-240. doi: 10.1038/13874

Ratkevicius, A., Carroll, A. M., Kilikevicius, A., Venckunas, T., McDermott, K. T., Gray, S. R., et al. (2010). H55N polymorphism as a likely cause of variation in citrate synthase activity of mouse skeletal muscle. Physiol. Genomics 42, 96-102. doi: 10.1152/physiolgenomics.00066.2010

Shao, H., Burrage, L. C., Sinasac, D. S., Hill, A. E., Ernest, S. R., O’Brien, W., et al. (2008). Genetic architecture of complex traits: large phenotypic effects and pervasive epistasis. Proc. Natl. Acad. Sci. U.S.A. 105, 19910-19914. doi: 10.1073/pnas.0810388105

Singer, J. B., Hill, A. E., Burrage, L. C., Olszens, K. R., Song, J., Justice, M., et al. (2004). Genetic dissection of complex traits with chromosome substitution strains of mice. Science 304, 445-448. doi: 10.1126/science.1093139

Steiner, J. L., Murphy, E. A., McClellan, J. L., Carmichael, M. D., and Davis, J. M. (2011). Exercise training increases mitochondrial biogenesis in the brain. J. Appl. Physiol. 111, 1066-1071. doi: 10.1152/japplphysiol.003 43.2011
The Jackson Laboratory (2010). Echocardiography and Auditory Brainstem Response Survey in Males of 7 Inbred Strains of Mice. MPD:35025. Mouse Phenome Database Web site. Bar Harbor, ME: The Jackson Laboratory.

Vollaard, N. B., Constantin-Teodosiu, D., Fredriksson, K., Rooyackers, O., Jansson, E., Greenhaff, P. L., et al. (2009). Systematic analysis of adaptations in aerobic capacity and submaximal energy metabolism provides a unique insight into determinants of human aerobic performance. J. Appl. Physiol. 106, 1479-1486. doi: 10.1152/japplphysiol.91453.2008

Wang, Y. X., Zhang, C. L., Yu, R. T., Cho, H. K., Nelson, M. C., Bayuga-Ocampo, C. R., et al. (2004). Regulation of muscle fiber type and running endurance by PPARdelta. PLoS Biol. 2:e294. doi: 10.1371/journal.pbio.0020294

Zhang, S. J., Bruton, J. D., Katz, A., and Westerblad, H. (2006). Limited oxygen diffusion accelerates fatigue development in mouse skeletal muscle. J. Physiol. 572. 551-559. doi: 10.1113/jphysiol.2005.104521

Conflict of Interest Statement: The authors declare that the research was conducted in the absence of any commercial or financial relationships that could be construed as a potential conflict of interest.

Copyright (C) 2017 Kvedaras, Minderis, Fokin, Ratkevicius, Venckunas and Lionikas. This is an open-access article distributed under the terms of the Creative Commons Attribution License (CC BY). The use, distribution or reproduction in other forums is permitted, provided the original author(s) or licensor are credited and that the original publication in this journal is cited, in accordance with accepted academic practice. No use, distribution or reproduction is permitted which does not comply with these terms. 